\title{
New targets for treatment, prevention and control of carbapenem-resistant A. baumannii isolate causing nosocomial infections
}

\section{Introduction}

Acinetobacter spp. are among the most frequent causes of nosocomial bacterial infections. This genus have gained increasing attention as different members of the family show multiple virulence factors that cause clinical infections with high rates of mortality but also the possibility of industrial applications due to the production of some enzymes useful for bioremediation. ${ }^{1}$ Up to know 41 distinct validly named species, ${ }^{2}$ although there are a number of strains of provisional termed genomic species, several with not validly name and others of an unknown taxonomic status. ${ }^{3}$

Acinetobacter baumannii is undoubtedly the species showing the highest clinical relevance although during the last years $A$. pittii and A. nosocomialis (also known as the A. baumannii group) are also emerging as nosocomial pathogens. ${ }^{1,4,5}$ Two main features contribute to the great interest of $A$. baumannii over the other related species: first, the ability of clinical isolates to develop multidrug- resistance acquired by either mutations or genetic elements such as plasmids, transposons, or resistant islands and second, the ability to colonize almost any surface and survive in the environment making it very difficult to eradicate from clinical settings. ${ }^{1,4}$

During the last decades A. baumannii has emerged as one of the most dangerous pathogens and a priority of the World Health Organization. It has been currently added to the hit-list of most important hospital pathogens known as "ESKAPE". ${ }^{6}$ Clinical cases typically involve ventilator-associated pneumonia and septicaemia with high rates of mortality, and also endocarditis, meningitis, burn and surgical wound infections, and urinary tract infections. Bacteriemia is very common in elderly immunocompromised patients being pneumonia the main source of the majority of cases. Nosocomial pneumonia affects patients in intensive care units with a rate of mortality of 30 to $75 \% .{ }^{1,5}$ Risk factors to acquire Acinetobacter infections include major surgery, malignant diseases or burns, immunocompromised patients such as the elderly, neonates with low birth weights, and patients with prolonged illnesses. Predisposing factors are malignant diseases, trauma, prolonged antibiotic treatment, prolonged intravenous lines, post operations, urinary catheterization, mechanical ventilation, parenteral nutrition and long hospitalization.

The emergence of nosocomial infections caused by A. baumannii is a result of different factors:
a) a succesful adaptation to adverse environmental conditions
b) the long persistance on dry surfaces
c) the resistance to antibiotics
d) the increase of immunocompromised patients
e) plasticity of their genome

\section{Ecology}

Acinetobacter spp. are mostly free living saprophytes found ubiquitously in nature but the precise environmental reservoirs are
Volume 2 Issue 4 - 2015

\author{
Lucia Gallego \\ Department of Immunology, Microbiology \& Parasitology, \\ University of the Basque Country UPV/EHU, Spain
}

\begin{abstract}
Correspondence: Lucia Gallego, Antibiotics \& Molecular Bacteriology Laboratory, Department of Immunology, Microbiology \& Parasitology, Faculty of Medicine \& Dentistry, University of the Basque Country UPV/EHU, Spain, Barrio sarriena s/n, 48940 Leioa, Bizkaia, Spain, Tel +34-9460I2778, Fax+34-9460I2869, Email lucia.gallego@ehu.es
\end{abstract}

Received: August 7,2015 | Published: August 13, 2015

unknown (Doughary). Generally considered part of the normal flora of the skin and mucosa of the pharynx, human respiratory secretions, urine, rectum and other clinical samples. They are the only group of gram-negative bacteria that may be natural residents of the human skin with carriage rates of $42.5 \%$ in healthy individuals and as high as $75 \%$ in hospitalized patients. Generally the species most frequently isolated are A. johnsonii, A. lwoffii, A. radioresistens, A. baumannii, A. calcoaceticus, A. haemolyticus, A. pittii and A. nosocomialis. Colonization of the intestinal tract is controversial but some authors suggest and report that there is the most important reservoir of resistant strains. ${ }^{1,4}$

The A. baumannii has been reported to inhabit soil and aquatic environments. The organism has also been isolated from different sources such as freshwater ecosystems, raw sewage and wastewater treatment plants and activated sludge, vegetables, fruit and animal sources, it has also demonstrate its ability to grow on crude oil (Obuekwe) and it has also been isolated from lice collected from homeless people. Several studies have shown that strains of $A$. baumannii can adhere to human cells and form biofilm on abiotic surfaces what enhaces its capacity of survival en the environment (on fingertips and inanimate objects such as glass, plastic, and other environmental surfaces) even after exposure to dry conditions and nutrient starvation during extended periods of time. ${ }^{1,4}$

In the hospital environment $A$. baumannii is the most important species. It has been isolated from ventilator tubings, arterial pressure monitoring devices, humidifiers, washbasins and also from the skin of healthcare personnel, mattresses, pillows and in all types of ventilator equipment and moist situations such as sinks and tap water. Hospital food could also be a potential source of A. baumannii. Some strains also survive exposure to commonly used disinfectants like chlorhexidine, gluconate and phenols, particularly those not used in the appropiate concentrations and is able to survive much better, compared with other gram-negatives, on fingertips or on dry surfaces when tested under simulated environmental conditions. ${ }^{1,4,6}$ 
It is clear that the emergence of nosocomial and communityacquired infections due to A. baumannii is mainly a result of high adaptability to adverse environmental conditions and the ability to persist for months in dry and harsh environments. They are able to survive on moist and dry surfaces and some strains have been found to be tolerant of soap.

\section{Pathogenesis and virulence}

Multiple virulence factors have been described in A. baumannii but little information is known about the contribution to its pathogenicity. The pathogenic mechanisms are little understood or studied and the precise mechanisms involved in the establishment and progression of infections by this species are unclear. Among the most important pathogenic mechanisms and virulence factors are:

a) twitching motility mediated by a type IV pilus and adherence to biotic and abiotic surfaces due to the production of structures such as pili and forming biofilms

b) surface polysaccharides forming capsules, extracellular polysaccharidae (PNAG) and LPS structures

c) OmpA which induces the apoptosis in epithelial cells and degrades chromosomal DNA and is also implicated in resistance to complement and biofilm formation

d) Outer Membrane Vesicles containing multiple virulence factors (OmpA, proteases, phospholipases, LPS, liporpoteins, bacterioferritin, catalase, etc.)

e) Phospholipases, important for human serum resistance, epithelial cell invasion, etc

f) enhaced virulence in the presence of ethanol not only assimilated as carbon source but also due to increased expression of some factors related to stress responses

g) Penicillin-binding proteins; g] siderophores for iron uptake

h) quorum sensing systems related to production of biofilm and other virulence mechanisms

i) antimicrobial resistance to almost all commonly used antibiotics

j) genomic plasticity and versatility due to the diversity of genomic islands and mobile structures such as plasmids, integrons and IS $A b a$ elements. ${ }^{1,4}$

\section{Emergence of carbapenem-resistant clones causing nosocomial infections}

During the last years Acinetobacter baumannii has become resistant to almost all commonly used antimicrobial agents, including $\beta$-lactams, amynoglycosides and quinolones and isolates showing multidrug or pan-resistant phenotype are described in the clinical setting worldwide. In no organism is the antibiotic panresistant paradigm more evident than in Acinetobacter baumannii that has acquired resistance genes to virtually all antibiotics capable of treating Gram-negative bacteria. The continuing rise in the number of such isolates is of great concern as there are no new antibiotics development resulting most of the infections fatal. ${ }^{7,8}$

For resistant isolates, carbapenems are the last therapeutic option due to their good activity and low toxicity but resistance to this group of antibiotics has been increasing during the last years. Although alterations to PBPs, loss or decreased expression of OMPs and efflux systems can confer resistance to carbapenems, in Acinetobacter baumannii the most important mechanism is the production of carbapenemases. Several classes of beta-lactamses have been identificated in A. baumannii:

a) class $\mathrm{A}$ which includes the majority of the extended-spectrum beta-lactamases (ESBL) TEM, SHV and CTX-M families and GES and KPC carbapenemases

b) class B metallo-beta-lactamases of the IMP, VIM, SIM and NDM families

c) class $\mathrm{C}, \mathrm{ADC}$ enzymes (also referred to as $\mathrm{AmpC}$ enzymes)

d) class $\mathrm{D}$ beta-lactamases that are by far the most prevalent in $A$. baumanni. $^{7}$

There are five well known families of OXA-type carbapenemases that have been found in A. baumannii: the OXA-23-like, OXA-40like, OXA-51-like, OXA-58-like and OXA-143-like ${ }^{9}$ and a sixth group recently described and named OXA-235. ${ }^{10}$ All A. baumannii isolates possess an inherent class D beta-lactamase gene bla OXA- 51 but they can additionally produce more. While other carbapenemases are commonly found to be located on class 1 integrons, bla NMD and the OXA-type enzymes genes are frequently been associated with ISAba elements and located on plasmids which facilitates the overexpresion and transferability of the resistance genes. ${ }^{11}$ To add to this a number of resistance islands $A b a \mathrm{R}$ have been identified among multidrug-resistance isolates belonging to worldwide epidemic lineages.

Outbreaks of $A$. baumannii are often clonal and a large number of infections globally can be attributed to three of these lineages named WW1, WW2 and WW3 corresponding to the European clones I, II and III that are commonly resistant to carbapenems. ${ }^{11,12}$ The reason why some lineages are particularly successful and spread internationally is unclear but it's clear that the selective advantage that multiple antibiotic resistance confer, appears to be driving their expansion. While the core genome of these lineages is stable, the accessory genome is more fluid. . $^{3,13,14}$

\section{Future alternatives}

The cost to control outbreaks can be staggering, and some institutions have been forced to close units in order to interrupt the transmission of Acinetobacter. There is a need to prevent transmission in the healthcare setting and keep the organism, especially multidrugresistant isolates, from becoming endemic in an institution. To achieve this goal it is also important to control contamination of the environment, water or food. Careful personal and hand-hygiene should be observed. It is important that laboratories should embark on active surveillance to detect cultures and patients who are colonized with multidrug-resistant Acinetobacter as well as a community-based surveillance to determine carriage rates. Other measures successful in the control of outbreaks include isolation precautions for infected or colonized patients, patients' relatives and staff, environmental disinfection, antimicrobial control, and unit closure if needed.

Implementing control of WW clones is a priority as these multidrug-resistant clones serve as host for the multiple genetic elements (integrons, IS, pAs, transposons and plasmids) that confer their resistance phenotype. Strains belonging to these successful WW clones I, II and III are an effective vehicle for the dissemination of 
the elements vertically and acting as donors to transfer its resistance elements horizontally to other strains, species or genera. As the majority of isolates belonging to these clones locate the resistance genes on plasmids, identification of this structures and control of its transferability is needed..$^{3,13-16}$

Treatment of Acinetobacter infections should be individualized according to susceptibility patterns. Carbapenem resistance has been described as a global sentinel event making evident we need to implement effective intervention and control strategies. The ability of the OXA-type beta-lactamases to confer carbapenem resistance has had a huge impact on the ability to treat $A$. baumannii infections and the current situation suggests that the problem is set to increase. ${ }^{7,8,17}$ As preservation of carbapenem efficacy is essential for continued success against this microorganism, we need to be very careful in the management of carbapenems and promote the combination of these antibiotics with $\beta$-lactamase inhibitors which could overcome strains with these resistance. ${ }^{17,18}$

In summary, strategies searching for new classes of antibiotics and knowledge on pathogenicity mechanisms and virulence factors seemed to be promising. Research on novel antimicrobial agents does not seem very promising due to the ability of $A$. baumannii to develop a huge range of resistance mechanisms but investigation of novel antibiotic substances from natural sources should also be considered. The novel strategies to interfere with the pathogenicity and bacterial growth were opens up a challenging alternative. Some of these targets could be vaccines against PNAG, OmpA and OMV antigens, antibiotics against OmpA and interruption of quorum sensing signals for pathogenesis inhibition.

\section{Acknowledgments}

None.

\section{Conflicts of interest}

Author declares that there is no conflict of interest.

\section{References}

1. Doughari HJ, Ndakidemi PA, Human IS, et al. The Ecology, Biology and Pathogenesis of Acinetobacter spp.: an overview. Microbes Environ. 2011;26(2):101-112.

2. http://www.bacterio.net/acinetobacter.html

3. Touchon M, Cury J, Yoon EJ, et al. The genomic diversification of the whole Acinetobacter genus: origins, mechanisms, and consequences. Genome Biol Evol. 2014;6(10):2866-2882.
4. Roca I, Espinal P, Vila-Farres X, et al. The Acinetobacter baumanni oxymoron: commensal hospital dweller turned pan-drug-resistance menace. Front Microbiol. 2012;3:148.

5. Canton R, Ruiz-Garbajosa P. Acinetobacter baumannii: Do they still deserve our attention? Enferm Infecc Microbiol Clin. 2013;31(1):1-3. [Article in Spanish].

6. Boucher HW, Talbot GH, Bradley JS, et al. Bad Bugs, No Drugs: No ESKAPE! An Update from the Infectious Diseases Society of America. Clinical Infectious Diseases. 2009;48(1):1-12.

7. Evans BA, Hamouda A, Amyes SGB. The Rise of Carbapenem-Resistant Acinetobacter baumannii. Current Pharm Design. 2013;19(2):223-238.

8. Kempf M, Rolain JM. Emergence of resistance to carbapenems in Acinetobacter baumannii in Europe: clinical impact and therapeutic options. Int J Antimicrob. 2012;39(2):105-114.

9. Evans BA, Aymes SGB. OXA $\beta$-Lactamases. Clin Microbiol Rev. 2014;27(2):241-263.

10. Higgins PG, Perez-Llarena FJ, Zander E, et al. OXA-235, a novel class D beta-lactamase involved in resistance to carbapenems in Acinetobacter baumannii. Antimicrob Agents and Chemother. 2013;57(5):2121-2126.

11. Karah N, Sundsfjord A, Towner K, et al. Insights into the global molecular epidemiology of carbapenem non-susceptible clones of Acinetobacter baumannii. Drug Resist Updat. 2012;15(4):237-247.

12. Woodford N, Turton JF, Livermore DM. Multiresistant Gram-negative bacteria: the role of high-risk clones in the dissemination of antibiotic resistance. FEMS Microbiol Rev. 2011;35(5):736-755.

13. Diancourt L, Passet V, Nemec A, et al. The Population Structure of Acinetobacter baumannii: Expanding Multiresistant Clones from an Ancestral Susceptible Genetic Pool. PLoS One. 2010;5(4):e10034.

14. Fondi M, Bacci G, Brilli M, et al. Exploring the evolutionary dynamics of plasmids: the Acinetobacter pan-plasmidome. BMC Evol Biol. 2010;10:59.

15. Garcillan-Barcia MP, Alvarado A, de la Cruz F. Identification of bacterial plasmids bases on mobility and plasmid population biology. FEMS Microbiol Rev. 2011;35(5):936-956.

16. Towner KJ, Evans B, Villa L, et al. Distribution of intrinsic plasmid replicase genes and their association with carbapenem-hydrolyzing class D beta-lactamase genes in European clinical isolates of Acinetobacter baumannii. Antimicrob Agents Chemother. 2010;55 (5):2154-2159.

17. Carlet J, Jarlier V, Harbarth S, et al. Ready for a world without antibiotics? The pensieres antibiotic resistance call to action. Antimicrob Resist Infect Control. 2012;1(1):11.

18. Walsh F, Amyes SGB, Duffy B. Challenging the concept of bacteria subsisting on antibiotics. Int J Antimicrob Agents. 2013;41:558-563. 